“( 2014 IEEE. Personal use of this material is permitted. Permission from IEEE must be obtained for all other uses, in any current or future media, including

reprinting/republishing this material for advertising or promotional purposes, creating new collective works, for resale or redistribution to servers or lists, or reuse of any copyrighted component of this work in other works." 


\title{
Multi-Gigabit Microwave and Millimeter-Wave Communications Research at CSIRO
}

\author{
Xiaojing Huang, Y. Jay Guo, and Jian A. Zhang \\ CSIRO Digital Productivity and Services, Sydney, Australia \\ Emails: \{Xiaojing.Huang, Jay.Guo, Andrew.Zhang\}@csiro.au
}

\begin{abstract}
High speed and long range wireless backhauls are cost-effective alternatives to fibre networks and becoming more and more attractive as the demand for broadband wireless services grows rapidly in recent years. However, current commercially available wireless backhaul systems neither provide sufficiently high speed nor meet the requirements to achieve both high speed and long range at the same time with sufficiently low latency for targeted applications. Traditional microwave systems can achieve long transmission range, but the data rates are limited to a few hundred Mega bits per second only. Multi-Gigabit wireless communications can be achieved using millimetre-wave (mm-wave) frequency bands, especially the $\mathrm{E}$ bands, but the practical transmission range is still a major weakness. In this paper, the state-of-the-art microwave and $\mathbf{m m}$ wave technologies developed at the Commonwealth Scientific and Industrial Research Organization (CSIRO) are introduced to demonstrate CSIRO's technology leadership in multi-Gigabit wireless communications research and development. The technology trends in multi-Gigabit wireless communications are also discussed and various recently developed microwave and $\mathbf{m m}$ wave systems are compared. It is hoped that this paper will stimulate further research interest and industry development.

Index Terms-Gigabit wireless, microwave, mm-wave, backhaul, and low latency.
\end{abstract}

\section{INTRODUCTION}

According to the Cisco visual networking index (VNI) global mobile data traffic forecast [1], global mobile data traffic reached 1.5 Exabytes ( 1 Exabytes $=2^{60}$ bytes) per month at the end of year 2013, and is expected to grow to 15.9 Exabytes per month by 2018, which represents a compound annual growth rate (CAGR) of 61 percent. Such a rapid increase in mobile data traffic is mainly due to the widespread deployment of broadband wireless access (BWA) services enabled by high speed user terminals, such as smart phones and tablets, as well as the next generation BWA networks, such as the worldwide interoperability for microwave access (WiMAX) [2] and the third generation partnership project (3GPP) long term evolution (LTE)-Advanced [3] systems, which can offer 100 Mega bits per second (Mbps) to 1 Gigabits per second (Gbps) data rates. These high speed BWA networks serve as attractive alternatives to wired access networks such as fibre optic networks to provide broadband services in a cost-effective manner. In the meantime, network operators are seeking solutions to support low latency applications, such as real-time machine-to-machine communications, interactive multiplayer gaming, high frequency trading, and cloud computing [4].
Due to the ever increasing capacity required to support high speed broadband services, the backhaul network, which transfers data traffic from cell sites of a wireless access network to the core network or a switching centre, is under intensive pressure. There are a number of challenges posed to such backhauls by the emerging high speed BWA networks as well as other application specific data networks. The first one is how to achieve higher data rate or capacity up to multiple Giga bits per second. For example, if the capacity of a cell (or sector) in a BWA base station is 1 Gbps, the backhaul capacity required by a three sector base station would be at least 3 Gbps. Sometimes the traffic from multiple base stations will be aggregated together across a backhaul link before reaching the core network. This will drive the backhaul capacity to a much higher rate, say 10-15 Gbps. The second challenge is the link distance of the backhaul. To deliver BWA services to unserved areas, such as the rural and regional areas which are often quite remote from the main telecommunication infrastructure, a long distance backhaul link is required. In countries with large geographical areas of low population density, such as Australia, long distance high data rate wireless backhauling links are essential to bring broadband services to the last $10-30 \%$ of the population. The third challenge is how to achieve low latency communications between end users across the backhaul networks. Although low latency has always been important for delivering high quality voice, video and data services in broadband networks, recent application requirements within many industry sectors, such as gaming and finance, have brought low latency right to the forefront of the telecommunication industry.

Fibre is the primary medium to deliver leased synchronous digital services and Ethernet services. It is the first choice to offer high data rate backhauling form $155 \mathrm{Mbps}$ to $10 \mathrm{Gbps}$ capacity. However, due to the high installation expenses such as digging the trenches in which the fibre is to be laid, owning a fibre is a significantly more expensive capital expenditure (CAPEX) option. It is also estimated that leased lines currently account for roughly 15 percent of the network operating expenditure (OPEX). On the contrary, wireless backhaul is more cost-effective than leased T1/E1, DS3, or OC-3 lines. In addition to the economic benefits of ownership, wireless backhaul also allows service providers to retain end-to-end control of their data, gain the security, stability and freedom associated with the full control over their own network. For less populated rural areas, where the cost to lay fibre can be 
prohibitive, wireless backhaul will be the only viable solution. Finally, because radio propagates over the air faster than light travels through fibre, wireless backhaul can achieve lower endto-end latency than fibre.

Therefore, if wireless backhaul can achieve multi-Gigabit data rates comparable to fibre capacity, it will be a costeffective solution to fibre replacement in urban areas and a very attractive proposition for providing remote communities with broadband services. It can also serve as or be part of an ultra-low latency network for various low latency applications.

The rest of this paper is organized as follows. Firstly, CSIRO's innovative technologies to achieve high speed long range microwave backhaul and high rate low latency $\mathrm{mm}$ wave backhaul are introduced in Section II and Section III respectively. Then, current technology trends in achieving high speed wireless transmission are briefly reviewed in Section IV. A comparison among various recently developed microwave and $\mathrm{mm}$-wave systems is made in Section $\mathrm{V}$ to demonstrate CSIRO's technology leadership. Finally, conclusions are drawn in Section VI.

\section{CSIRO MICROWAVE BACKHAUL}

Microwave $(6-40 \mathrm{GHz})$ is the typical medium for wireless backhaul. The microwave spectrum is divided into specific bands administered by national regulators in individual countries. In Australia, such government agency is the Australian Communications and Media Authority (ACMA). Since the bandwidth of each microwave channel is narrow (7 to 55 $\mathrm{MHz}$ ) [5], the data rates of traditional microwave links are very low, up to several hundred Mbps. To achieve higher speed, sufficient bandwidth is necessary. However, a large contiguous bandwidth is not available at microwave frequencies for backhauling, except for some disjoint radio frequency (RF) bands and channels. A natural solution is to aggregate multiple channels to obtain the required bandwidth as has been implemented in some vendors' products. However, such a straightforward combination of multiple low rate wireless systems is neither cost-effective nor spectrally efficient. On the other hand, due to the radio propagation characteristics, the radio frequencies should be below $10 \mathrm{GHz}$ for long range operation. Achieving both high speed and long range for wireless backhauls remains as a significant technical challenge.

CSIRO has recently developed a multi-Gigabit long distance microwave backhaul system [6], called Ngara backhaul. The system employs a number of novel approaches to realize high rate wireless transmission. The key innovations include the aggregation of multiple RF channels in multiple frequency bands digitally to increase transmission bandwidth and spectral efficiency [7], a novel peak-to-average power ratio (PAPR) reduction by using single carrier frequency division multiple access (SC-FDMA) to increase power efficiency, a novel outof-band emission cancellation technique to suppress interference to adjacent unallocated RF channels [8], and software defined radio (SDR) system architecture [9] to reduce the number of signal processing chains, RF chains and hence the overall implementation cost.

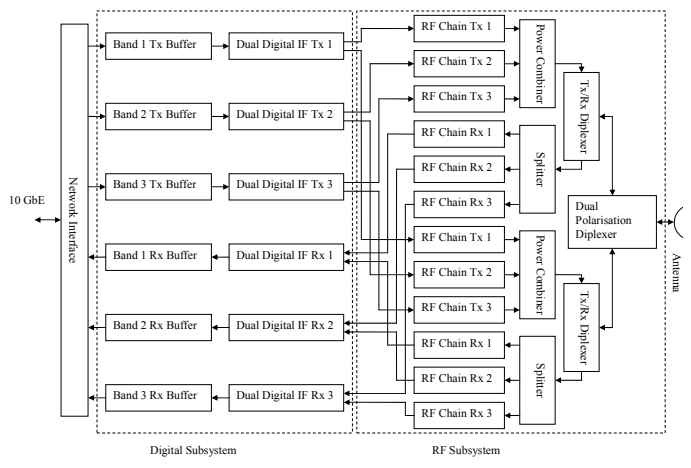

Fig. 1. Multi-band microwave transceiver block diagram.

The microwave frequency bands at $6,6.7$, and $8 \mathrm{GHz}$ have been selected for achieving long range operation of the Ngara backhaul. Scalable multi-Gigabit wireless link can be realized by aggregating certain number of RF channels in these bands to obtain sufficient bandwidth in these frequency bands. For example, if all the RF channels in the three bands are aggregated together, the total bandwidth will be over $724.75 \mathrm{MHz}$. If multilevel modulation such as 256 quadrature amplitude modulation (QAM) is used, a spectral efficiency of $6.95 \mathrm{bps} / \mathrm{Hz}$ (including signalling overhead) can be achieved and the data rate will be over 5 Gbps. By further employing dual polarisation, a $10 \mathrm{Gbps}$ wireless link with $13.9 \mathrm{bps} / \mathrm{Hz}$ spectral efficiency can be achieved.

A system block diagram of the multi-band transceiver is shown in Fig. 1, which consists of a network interface, a digital sub-system, and an RF sub-system. The digital sub-system generates and receives digital intermediate frequency (IF) signals in different frequency bands. The RF sub-system converts IF signals to/from RF bands, amplifies and transmits/receives RF signals. In each RF chain of a frequency band, analogue anti-aliasing filters (AFs) are used to eliminate the unwanted Nyquist responses and image responses respectively. Bandpass filters (BPFs) are used to select signals from the frequency band after/before the up/down conversion with an appropriate local oscillator (LO) frequency. For transmitter (Tx), the upconverted RF signal in each frequency band is amplified by a power amplifier (PA) and combined with amplified RF signals from other bands. For receiver (Rx), the received RF signal is passed through a splitter and then amplified by a low noise amplifier (LNA) in each bands. This transceiver implementation represents a feasible technical solution for which most of the RF modules such as PAs and LNAs can be built using commercial-off-the-shelf (COTS) microwave components. However, the RF filters, diplexer, efficient power combiner and wideband antenna feed are non-trivial and need to be developed in house.

\section{CSIRO MM-WAVE BACKHAUL}

Multi-Gigabit mm-wave communications systems are gaining increasing interest and importance to the wireless industry recently. Typical mm-wave bands suitable for wireless backhaul application include $60 \mathrm{GHz}$ band and 70/80 GHz E-band. 
Commercial point-to-point links in the $60 \mathrm{GHz}$ band with data rates up to $1.25 \mathrm{Gbps}$ are available from several manufacturers. However, high propagation loss due to oxygen absorption in this band and regulatory requirements limit the communication range for outdoor applications to $0.5-0.8 \mathrm{~km}$ only. The recent availability of the E-band spectrum worldwide provides an opportunity for line of sight (LOS) links with longer range and higher data rates, ideally suited for fibre replacement and backhaul applications. The spectrum with bandwidth over 5 $\mathrm{GHz}$ available in the E-bands allows for multi-Gigabit data rates. The merits of E-band wireless communications include the vast, uncongested, inexpensive spectrum with total $10 \mathrm{GHz}$ of available RF bandwidth which enables very high data rates beyond $10 \mathrm{Gbps}$, the small highly directional antenna, low output power, and low infrastructure cost. These advantages make the E-band links ideally suited for short range (1-5 $\mathrm{km})$ fibre quality wireless communications. Current E-band commercial point-to-point wireless links (e.g., available from BridgeWave, LOEA, Proxim, E Band Communications, Elva, and others) are limited to speeds up to $1.25 \mathrm{Gbps}$ and use simple modulation techniques like amplitude shift keying (ASK) or binary phase shift keying (BPSK) with spectral efficiency below one bps/Hz.

Technologies enabling much higher data rates (up to 24 Gbps) were developed and reported several years ago by the CSIRO ICT Centre [10]. The key method developed by CSIRO is applicable to systems where the radio channel bandwidth is greater than the Nyquist bandwidth of the associated analogueto-digital converter (ADC) and digital-to-analogue converter (DAC). Such systems can be utilized for the E-band fullduplex wireless links with a spectral efficiency scalable from 2.4 to $4.8 \mathrm{bit} / \mathrm{s} / \mathrm{Hz}$ for 8 phase shift keying (PSK) to 64QAM modulations to transmit 12 to $24 \mathrm{Gbps}$. This has been proven by experimental results on a 6 Gbps prototype with spectral efficiency of $2.4 \mathrm{bps} / \mathrm{Hz}$.

A simplified block diagram of the mm-wave backhaul system is shown in Fig. 2. The system includes a network interface, a digital modem including multiplexing and demultiplexing, an IF module, and a wideband mm-wave frontend with a high-directivity antenna. The transmit and receive signals are combined using either a frequency or time domain diplexer. At the transmitter input, the digital data stream is demultiplexed into $\mathrm{N}$ digital channels (e.g., $\mathrm{N}=4$ to 16 ). Each digital channel is generated by a field programmable gate array (FPGA) and a high-speed DAC. At the modulator, several identical high data rate digital channels are generated by direct computation of the analogue frequency signals. Analogue IF signals of each digital channel are multiplexed in the frequency domain in a spectrally efficient manner without a need of the frequency guard bands between the adjacent channels. The combined IF signal is up-converted into an mm-wave carrier frequency amplified by a PA and transmitted over a LOS path. At the receiver, the received signal is passed through a BPF and LNA and down-converted from the mm-wave carrier frequency into IF and demultiplexed in the frequency domain into multiple channels, then sampled by the high speed ADCs

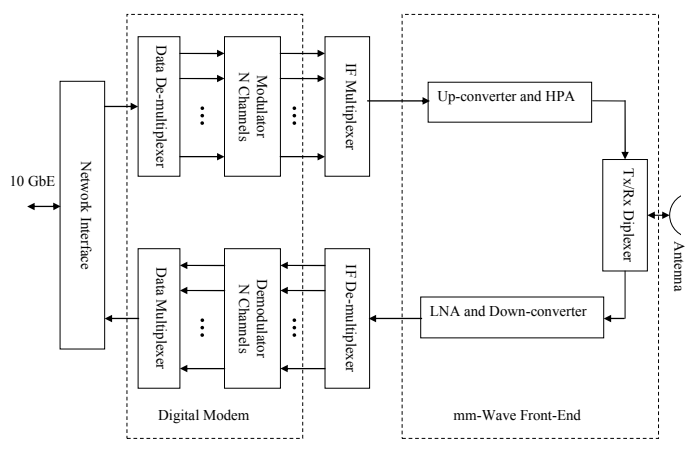

Fig. 2. High spectral efficiency mm-wave transceiver block diagram.

and decoded by the FPGA into digital channels and then multiplexed into a single digital stream.

With the advance in mixed signal processing technologies, higher speed ADC and DAC and larger scale FPGA devices are now available. The spectral efficiency of above E-band system based on frequency-domain multiplexing can be further improved. Combined with dual polarisation, a high speed Eband link of up to $50 \mathrm{Gbps}$ data rate can be achieved in 5 $\mathrm{GHz}$ bandwidth.

Because radio propagates over the air faster than light travels through fibre, wireless link can achieve lower latency than fibre network. This allows for E-band wireless links to replace fibre network for low latency applications such as high frequency trading with comparable data rate. Even though the operation range per hop of the E-band link is limited, the endto-end latency of a multi-hop E-band system can be still lower than fibre network if the processing delay at radio relay nodes is only a fractional part compared with the propagation delay. Furthermore, with fibre link as a backup and operating only under favorable weather conditions, the per-hop distance of an E-band link can be extended to over $20 \mathrm{~km}$. This will further reduce the overall end-to-end latency and deployment cost for the multi-hop E-band link.

Targeted at the low latency applications, CSIRO has developed a high speed (up to $10 \mathrm{Gbps}$ ) E-band system with processing delay less than 0.5 microsecond and operation distance over $20 \mathrm{~km}$ per hop. A different architecture from the one described above has been adopted and a number of novel techniques have been developed in order to achieve high power efficiency and low latency. The major architectural difference is the use of in-phase and quadrature (I/Q) modulation at the IF stage rather than digital IF. Also, the $5 \mathrm{GHz}$ bandwidth is used as a single channel rather than being divided into multiple sub-channels. The novel techniques include advanced channel estimation and equalization combined with I/Q imbalance estimation and compensation, efficient transmitter and receiver filter design and implementation, and overall system optimization in terms of achieving both low latency and high performance at the same time.

Compared with other commercially available high speed Eband radios, CSIRO's low latency E-band system has some distinctive advantages. First, it has low processing latency 
due to the smart signal processing architecture and signalling protocol even though complicated error correction coding, equalisation, and practical impairment compensation algorithms are employed. Second, it can achieve $10 \mathrm{Gbps}$ data rate with relatively low level modulation (QPSK and 16 QAM) so that it offers higher power efficiency and hence longer distance. Third, with its advanced channel estimation and equalisation techniques, it can cope with harsh channel conditions with tens of nanoseconds delay spread caused by analogue circuitry, cable reflection, and multipath propagation. Finally, it offers flexible switching between Ethernet traffic and ultra-low latency relay traffic and among multiple radios so that multi-hop E-band link can be configured with fibre backup, suitable for low latency application under all weather conditions.

\section{TECHNOLOGY TRENDS}

On striving to further improve the wireless communications capacity and distance, significant efforts have been made in both the wireless industry and research community. There are a number of possible ways to achieve such improvements, which include employing higher order modulation, making use of LOS multiple input multiple output (MIMO) antennas, improving link availability, and increasing transmit power.

On employing higher order modulation, 1024QAM has been seen in commercial microwave product demonstration. Due to the DAC/ADC speed and resolution as well as phase noise issues, such higher order modulation can only be possible for narrow bandwidth (below $100 \mathrm{MHz}$ ) systems. For wider bandwidth systems in mm-wave bands, the highest modulation used so far is only 64QAM [11]. On the other hand, employing higher order modulation incurs some system penalties, such as design and implementation cost, reduced receiver sensitivity, i.e., higher signal-to-noise ratio (SNR) is required, and reduced output power due to linearity requirement in the power amplifier. The reduction in both transmit power and receiver sensitivity will lead to reduced link distance.

The use of LOS MIMO in microwave system has been recently demonstrated by Ericsson. By combining a 2x2 LOS MIMO with dual polarisation and 1024QAM, a spectral efficiency of $36 \mathrm{bps} / \mathrm{Hz}$ can be achieved, leading to $1 \mathrm{Gbps}$ throughput in a $28 \mathrm{MHz}$ channel [12]. Similar announcement has been made by MIMOtech that it has launched Starburst Janus, an ultra high capacity packet radio for last mile backhaul, which utilizes a $4 \times 4$ LOS MIMO yielding a spectral efficiency of $25 \mathrm{bps} / \mathrm{Hz}$ [13]. As a promising technology for future high speed wireless communications, LOS MIMO has also been proposed by the US Defense Advanced Research Projects Agency (DARPA) in its $100 \mathrm{G}$ program (DARPABAA-13-15) to develop a 100 Gbps RF backbone using Eband $\mathrm{mm}$-wave frequency spectrum.

Improving link availability is also an effective way to increase the capacity of the wireless communications, especially for mm-wave systems. Automatic transmit power control (ATPC) has been widely used in microwave radios but has not been widely adopted in mm-wave systems. By
ATPC, the transmit power can be deliberately reduced during clear air to reduce the risk of interference to nearby wireless systems, whereas during a rain fade the system automatically increases transmit power to the maximum level, allowing the link to overcome the fading effects. Other techniques include adaptive coding and modulation (ACM) and adaptive rate (AR). Although ACM has been widely adopted by wireless communications community, it may not applicable for all types of data traffic. It is still a challenge to cope with unpredictable and perhaps rapidly varying data rates. For mm-wave radios which only operate with low order modulations, AR may be a better solution. By taking the advantage of the large bandwidth, AR can keep the modulation constant but reduce the data rate during rain fades through reducing the bandwidth (and hence the noise floor).

Increasing transmit power is the most straightforward way to increase the operation distance. However, it may have both regulatory and technical issues. Technically, trying to increase individual PA output power continues to be one solution especially for microwave systems. For mm-wave systems, using antenna array with beamforming will be another potential solution, where each antenna elements can have low power. In order to achieve long range mm-wave communications in the E-band, CSIRO has developed a hybrid adaptive array technology $[14,15]$ and built a $4 \times 1$ array prototype. Although there are still a number of challenges, such as MMIC integration, efficient digital beamforming algorithm, and mutual coupling compensation, using adaptive array is a future research direction for over $200 \mathrm{~km}$ range inter-aircraft, aircraft-to-base station, and aircraft-to-vehicle communications.

As the E-band wireless systems become more and more popular for use in broadband networks with macro and small cells, it is expected that the E-band spectrum will be more and more congested. Unlike the unchannelized band plan in the US, each E-band in Europe has been divided into nineteen 250 $\mathrm{MHz}$ channels with a $125 \mathrm{MHz}$ guard band at the two sides of each $5 \mathrm{GHz}$ band in order to prevent possible interference from different E-band systems. Different channel combination and arrangement for time domain duplexing (TDD) and frequency domain duplexing (FDD) applications are also defined [16]. Even smaller bandwidth channels such as $62.5 \mathrm{MHz}$ have been recommended to allow more available links and more efficient spectrum usage. With such frequency allocation plan and increasing demand for E-band links, the use of narrower bandwidth and higher order modulation to offer multi-Gigabit data rate has become an industry trend for E-band system development. Therefore, E-band tends to be used in the way similar to conventional microwave bands.

\section{SYSTEM PERFORMANCE COMPARISON}

To summarise the pros and cons or the trade-offs among the various microwave and mm-wave communications techniques and demonstrate CSIRO's technology leadership, a performance comparison using the systems mentioned above as examples is provided in Table 1 . 
TABLE I

Performance Comparison among VARIOUs MicrowaVe AND Mm-WaVe TeChNologies

\begin{tabular}{|c|c|c|c|c|c|c|c|}
\hline \multirow{3}{*}{ Performance Metric } & \multicolumn{3}{|c|}{ Microwave } & \multicolumn{4}{|c|}{ mm-Wave } \\
\hline & Typical & CSIRO & Ericsson & Typical & Latest & CSIRO & CSIRO/EM Solutions \\
\hline & Commercial & Ngara & LOS MIMO & Commercial & Commercial & Low Latency & Next Generation \\
\hline Manufacturer & Aviat & CSIRO & Ericsson & BridgeWave & Huawei & EM Solutions & EM Solutions \\
\hline Model & Eclipse ODU300 & Research prototype & Demonstrator & FlexPort80 & RTN380(2nd Gen) & Product prototype & Product prototype \\
\hline Frequency Band(GHz) & $5-38$ & $6,6.7$, and 8 & Not available & 80 & $70 / 80$ & $70 / 80$ & $70 / 80$ \\
\hline Typical Bandwidth(MHz) & $7-56$ & 724.75(Aggregated) & 28 & 250 and 1000 & 250 & 4250 & Up to 5000 \\
\hline Modulation & QPSK to 256QAM & BPSK to 256QAM & 1024QAM & BPSK and QPSK & 64QAM & 16QAM & Up to 128QAM \\
\hline Maximum Data Rate(Mbps) & 360 & 10000 & 1000 & 1250 & 1250 & 10000 & 10000 \\
\hline Spectral Efficiency(bps/Hz) & 6 & 13.9 & 36 & 2 & 5 & 2.4 & 6 \\
\hline Processing Latency $(\mu \mathrm{S})$ & Not available & $<10$ & Not available & Not available & Not available & $<0.5$ & $<1$ \\
\hline Transmit Power(dBm) & Not available & 40 & Not available & Not available & Not available & 24 & 24 \\
\hline Operation Range $(\mathrm{km})$ & Not available & $>30$ & Not available & $1-2.5$ & $1-2.5$ & $>20$ & $1-20$ \\
\hline Cost and Complexity & Medium & High & High & Low & Medium & Medium & Medium \\
\hline Applications & General backhaul & High speed backhaul & Short range backhaul & Short range backhaul & Short range backhaul & Low latency backhaul & High speed backhaul \\
\hline
\end{tabular}

For microwave systems, CSIRO's Ngara backhaul is the only one which can offer $10 \mathrm{Gbps}$ data rate over long distance. Since it is a research prototype, the cost and complexity is relatively high at present. However, as the technology become mature and if the digital modem can be implemented by application specific integrated circuits (ASIC), the cost can be significantly reduced. High level modulation and LOS MIMO can significantly improve the spectral efficiency, however, since data rate also depends on channel bandwidth, it will be still difficult to produce high data rate without sufficient bandwidth. In addition, as higher level modulation reduces power efficiency, the operation range will be extremely limited.

For mm-wave systems, CSIRO's high spectral efficiency and low latency systems can offer the highest data rates with low latency. If combined with dual polarisation and LOS MIMO, the data rate can be increased beyond $100 \mathrm{Gbps}$ for the high spectral efficiency system. Also, the low latency system can be redesigned to have narrower bandwidth with higher level modulation, and thus provide both high data rate and low latency with significantly reduced cost. This low cost version of the low latency system will be very competitive for general short range mobile backhaul applications with distinct low latency advantage.

\section{CONCLUSiOnS}

With the advance of broadband wireless access and next generation mobile systems, huge demands are being placed on backhaul infrastructure. As cost-effective alternatives to fibre backhaul, high speed and long distance wireless backhauls are becoming increasingly attractive. However, there are significant technical challenges such as how to achieve higher spectral efficiency and extend transmission range. For long range wireless backhaul, the major challenge is to achieve multi-Gigabit data rate at microwave frequencies. With CSIRO's innovative Ngara backhaul technologies, the multiband microwave backhaul can provide up to $10 \mathrm{Gbps}$ data rates over several tens of kilometres range, making it particularly desirable for bringing high speed broadband services to rural and remote areas. The availability of the $10 \mathrm{GHz}$ spectrum in E-band provides an opportunity for high capacity wireless link, ideally suited for fibre replacement and backbone networks. CSIRO has solutions to tackle these challenges and is developing the most advanced E-band systems for high capacity low latency wireless applications.

\section{REFERENCES}

[1] Cisco, "Cisco Visual Networking Index: Globe Mobile Data Traffic Forecast Update, 2013-2018," white paper, available: http://www.cisco.com/c/en/us/solutions/collateral/serviceprovider/visual-networking-index-vni/white_paper_c11-520862.pdf

[2] I.-K. Fu et al., "Multicarrier Technology for 4G WiMAX System," IEEE Communications Magazine, vol. 48, no. 8, August 2010, pp. 50-58.

[3] S. Parkvall et al., "Evolution of LTE toward IMT-Advanced," IEEE Communications Magazine, vol. 49, no. 2, February 2011, pp. 84-91.

[4] Transmode, "Low Latency - How low can you go?" white paper, available: http://www.transmode/en/ resources/whitepapers

[5] Australian Communications and Media Authority (ACMA), "Microwave Fixed Services Frequency Coordination," 2008, available: http://www.acma.gov.au/webwr/radcomm/frequency_planning/frequency assignment/docs/fx $3 /$

[6] X. Huang, Y. J. Guo, J. Zhang, and V. Dyadyuk, "A Multi-Gigabit Microwave Backhaul," IEEE Communications Magazine, vol. 50, no. 3, March 2012, pp. 122-129.

[7] X. Huang, B. Murray, J. Bunton, Y. J. Guo, V. Dyadyuk, "Wireless Data Communications". Australian Provisional Patent (AU2009903399P) (July 2009), PCT published (WO 2011/009157 A1) (January 2011).

[8] J. Zhang, X. Huang, T. Cantoni, and Y. J. Guo, "Sidelobe Suppression with Orthogonal Projection for Multicarrier Systems," IEEE Transactions on Communications, vol. 60, no. 2, February 2012, pp. 589-599.

[9] X. Huang, Y. J. Guo, and J. Zhang, "Sample Rate Conversion Using BSpline Interpolation for OFDM Based Software Defined Radios," IEEE Transactions on Communications, vol. 60, no. 8, August 2012, pp. 21132122.

[10] V. Dyadyuk, J. Bunton, J. Pathikulangara, R. Kendall, O. Sevimli, L. Stokes, D. Abbott, "A Multi-Gigabit mm-Wave Communication System with Improved Spectral Efficiency". IEEE Transactions on Microwave Theory and Techniques, vol. 55, no. 12, December 2007, pp. 2813-2821.

[11] Huawei, "Huawei Debuts 2nd-Generation Ultra-Broadband EBand Microwave," Press Release, 2 October 2012, available: http://www.huawei.com/en/about-huawei/newsroom/press-release/hw194598-e-band microwave.htm

[12] J. Hansryd and J. Edstam, "Microwave Capacity Evolution," Ericsson Review, June 2011, available: http://www.ericsson.com/news/110621_microwave_capacity_evolution _244188810_c

[13] Cellular 4G/LTE Channel/Industry News, "MIMOtech Launches Ultra High Capacity Radio for Last Mile LTE Backhaul," Microwave Journal, 20 May 2013, available: http://www.microwavejournal.com/ articles/19885?utm_source=Microwave+Flash_Newsletter_20130522\&utm medium=OMAIL

[14] Y. J. Guo, J. Bunton, V. Dyadyuk, and X. Huang, "Multi-Stage Hybrid Adaptive Antennas," Australian Provisional Patent, AU2009900371 (2 February 2009), PCT published (20 August 2010) WO 2010/085854 A1.

[15] X. Huang, Y. J. Guo, and J. Bunton, "A Hybrid Adaptive Antenna Array," IEEE Transactions on Wireless Communications, vol. 9, no. 5, May 2010, pp. 1770-1779.

[16] ITU-R, "Radio-Frequency Channel and Block Arrangements for Fixed Wireless Systems Operating in the 71-76 and 81-86 GHz Bands," Recommendation ITU-R F.2006, March 2012. 